\title{
LA INFLUENCIA DE LOS PROFESIONALES SANITARIOS EN EL RECONOCIMIENTO Y EL DESARROLLO DE LA INTERPRETACIÓN MÉDICA
}

\author{
Almudena Nevado Llopis \\ anevado@usj.es \\ Universidad San Jorge, Zaragoza
}

\section{Resumen}

Numerosos estudios demuestran que las barreras lingüísticas a las que se enfrentan los profesionales sanitarios al comunicarse con los pacientes alófonos disminuyen la calidad de la asistencia prestada. Sin embargo, en los servicios sanitarios españoles con frecuencia se infravaloran estas dificultades comunicativas y se recurre a la interpretación ad hoc para tratar de superarlas. El propósito principal de este artículo consiste en mostrar cómo el desconocimiento por parte de los profesionales de la salud sobre la complejidad del rol del intérprete y sobre los riesgos que conlleva el empleo de intérpretes sin formación contribuye a que se recurra a estos últimos. Asimismo, en última instancia, pretendemos resaltar la influencia que ejercen los profesionales sanitarios en el desarrollo de la interpretación médica.

\begin{abstract}
"The influence of healthcare professionals on medical interpreting recognition and development"

The linguistic barriers that healthcare professionals encounter when communicating with foreign language-speaking patients diminish the quality of medical care, as has been demonstrated in several studies. Nevertheless, in Spanish health services, these communicative difficulties are frequently underestimated and, in order to overcome them, ad hoc interpreters are used. The main objective of this article consists in showing that the use of untrained interpreters partly derives from healthcare professionals' unfamiliarity with the complexity of the interpreters' role and their lack of awareness
\end{abstract}


of the risks involved in communicating through unqualified interpreters. Ultimately we expect to emphasize the influence of healthcare professionals on the development of medical interpreting.

Palabras clave: Profesionales sanitarios. Interpretación médica. Interpretación ad hoc. Interpretación telefónica.

Keywords: Healthcare professionals. Medical interpreting. Ad hoc interpreting. Telephone interpreting.

Manuscript received on January 29, 2013 and accepted for publication on December 20, 2013. 


\section{Contextualización}

La salud es un derecho humano universal del que deberían gozar todos los ciudadanos de las actuales sociedades multiculturales. Sin embargo, dicho derecho se vulnera en las situaciones en las que el paciente y el profesional sanitario, por no compartir la misma lengua, se encuentran con barreras que dificultan o impiden su comunicación. Estas barreras obstaculizan el acceso y uso de los servicios sanitarios por parte de los pacientes alófonos y, en definitiva, reducen la calidad de la asistencia que estos reciben. De hecho, diferentes estudios demuestran que la falta de una comunicación eficaz en el ámbito de la salud puede derivar en diagnósticos inapropiados, falta de adherencia al tratamiento, control o seguimiento del paciente deficientes, exámenes incompletos, o analíticas e ingresos hospitalarios innecesarios (Leanza 2007: 11-12).

En resumen, como sostiene Wadensjö (1998: 51), si los profesionales sanitarios no logran comunicarse con sus pacientes, si no son capaces de entender las explicaciones de estos ni de hacer entender las propias, difícilmente podrán ofrecer una adecuada asistencia sanitaria.

Entre las diferentes estrategias adoptadas para superar las mencionadas barreras, se encuentra el recurso a servicios lingüisticos, los cuales pueden ser proporcionados, en líneas generales, por cuatro tipos de figuras (Phelan \& Parkman 1995: 555): profesionales sanitarios bilingües, intérpretes profesionales, familiares o amigos del paciente, e intérpretes voluntarios o no profesionales.

Dejando de lado la figura de los profesionales bilingües, por alejarse de los fines que perseguimos en este artículo, el empleo de intérpretes profesionales es, sin duda, la vía más adecuada del resto de las planteadas para facilitar la comunicación entre el personal sanitario y los pacientes alófonos, ya que, al menos en teoría, esta figura ofrece la garantía de que posee las competencias mínimas para ejercer su labor y de que se regirá por los principios éticos inherentes a su profesión.

En cuanto a la tercera y la cuarta estrategias propuestas, podríamos englobarlas dentro de una misma categoría, en la que incluiríamos a cualquier persona que realiza labores de interpretación sin disponer de los conocimientos, 
habilidades y aptitudes necesarios para desempeñarlas con éxito. A este tipo de ejercicio se le suele denominar interpretación ad hoc o natural y, como veremos más adelante, son numerosas las consecuencias negativas que el recurso a la misma puede acarrear.

Por lo que se refiere a la adopción de una u otra figura, de acuerdo con Ozolins (2010), los países receptores de inmigración han dado cuatro tipos de respuestas distintas para superar las barreras lingüísticas que se plantean en la comunicación entre los profesionales de los servicios públicos (y, por consiguiente, de los servicios sanitarios) y los usuarios alófonos, las cuales consisten básicamente en la negación de la necesidad de proporcionar servicios de interpretación en el sector público (p.e., en algunos países de Europa del Este), la provisión de servicios ad hoc (p.e., en Italia), la creación de servicios lingüísticos genéricos (p.e., en Canadá) y la implantación de soluciones globales e integrales (p.e., en Reino Unido o en Australia).

En el contexto español destacan algunas iniciativas locales consistentes en el empleo de intérpretes profesionales en el ámbito de la salud, entre las cuales cabe mencionar la experiencia de algunos hospitales pioneros que han implantado servicios lingüísticos para favorecer la comunicación con los pacientes alófonos, como el Hospital Ramón y Cajal de Madrid o el Hospital Punta Europa de Algeciras, por citar algunos. Sin embargo, diversos estudios ${ }^{1}$ demuestran que la estrategia más comúnmente adoptada en España consiste en el empleo por parte de los hospitales y centros de salud de intérpretes ad $h o c$, a los que raramente se les exige que prueben su formación y preparación para ejercer su labor. El único criterio para seleccionarlos es frecuentemente el conocimiento personalmente declarado por el futuro intérprete de las dos lenguas de trabajo. Por supuesto, su remuneración y reconocimiento son en estos casos equivalentes y, por tanto, tan pobres como los criterios aplicados para su selección. Por este motivo, a menudo las personas con formación que podrían desempeñar adecuadamente esta actividad pierden el interés por ejercer en este ámbito y se dedican a la interpretación en otros contextos. En suma, en el ámbito sanitario español, tal y como señala Valero-Garcés (2013: 81), existe un desconocimiento generalizado de los beneficios que conlleva el recurso a la interpretación profesional para facilitar la comunicación con los pacientes lingüísticamente diversos, así como del alto riesgo y los costes que supone recurrir a soluciones ad hoc.

1. Valgan como ejemplo los estudios llevados a cabo por el grupo de investigación FITISPoS, por lo que se refiere a la zona centro (Madrid y alrededores), o los desarrollados por el Grupo MIRAS, por lo que respecta a Cataluña, y por el Grupo CRIT, en relación con la Comunidad Valenciana. 
De acuerdo con Corsellis (2010: 12-14), el avance de la interpretación en los servicios públicos (o, en nuestro caso, en los servicios sanitarios) puede atribuirse a las actitudes y comportamientos de tres grupos interdependientes. En primer lugar, son los propios intérpretes los que deberían luchar para que se estableciera un marco que regulara su desempeño profesional, contribuyendo, de este modo, a que se reconociera su formación y su experiencia y a que se les concediera el valor (en todos los sentidos, tanto social como económico) que se merecen. Otro de los grupos de los que depende la profesionalización de la interpretación en este ámbito está constituido por los gobiernos, responsables en última instancia de establecer estructuras y sistemas nacionales para la contratación de intérpretes. Asimismo, son los propios servicios sanitarios y, más concretamente, los profesionales que trabajan en ellos, los que deberían reconocer las barreras lingüísticas a las que se enfrentan a la hora de comunicarse con los pacientes alófonos y tratar de hallar una solución lo más adecuada posible para superar dichas barreras.

Cabe señalar que serán los dos últimos grupos mencionados los que ejerzan una mayor influencia a la hora de desarrollar esta profesión que, en palabras de Ozolins (2000, citado en Valero-Garcés \& Martin 2008: 4), es institution-driven. Por consiguiente, resulta necesario concienciar a dichos grupos y justificar, mediante investigaciones rigurosas, que destinar fondos públicos a mejorar la comunicación entre los profesionales de los servicios sanitarios y los pacientes que no hablan la lengua mayoritaria supondrá un beneficio para todos los implicados. De este modo, se fomentará el interés de los gobiernos y de los profesionales del ámbito de la salud por establecer una estructura coherente a nivel nacional que fomente la contratación de intérpretes debidamente cualificados y determine las competencias que estos deben poseer para realizar una interpretación de calidad.

\section{Objeto de estudio y metodología}

Por la imposibilidad de abordar en estas páginas el caso español en su conjunto, en el próximo apartado, a modo de ejemplo, describimos las estrategias y soluciones para eliminar las barreras comunicativas entre los profesionales sanitarios y los pacientes alófonos implementadas en los últimos años en los centros de salud de una ciudad española determinada, en concreto, localizada en la comunidad autónoma de Aragón.

Nuestro propósito principal consiste en tratar de demostrar el hecho de que factores como la negación o la infravaloración de las barreras lingüísticas por parte de los profesionales sanitarios, así como su desconocimiento sobre la complejidad del rol del intérprete y sobre los riesgos que conlleva el empleo 
de intérpretes ad hoc contribuyen a que se recurra a estos últimos, aun cuando existen servicios de interpretación profesional disponibles. Asimismo, pretendemos resaltar la influencia que pueden ejercer los profesionales de la salud en el desarrollo de la interpretación médica.

Los datos que presentamos proceden de una investigación en la que se analizó la comunicación entre los profesionales sanitarios y las usuarias de origen extranjero de un hospital materno-infantil de la mencionada ciudad. En particular, exponemos parte de los resultados obtenidos mediante, por una parte, la técnica del análisis documental ${ }^{2}$ y, por otra, la realización de veinticinco entrevistas personales semi-estructuradas y un grupo de discusión en los que participaron profesionales sanitarios del hospital contexto de estudio.

Las entrevistas fueron llevadas a cabo entre mayo y octubre de 2012. Con el fin de garantizar la representatividad de los informantes, estos ocupaban diferentes perfiles profesionales. En concreto, se entrevistó a cinco anestesistas, seis enfermeros, siete matronas, cuatro obstetras, y tres miembros del personal socio sanitario (dos auxiliares de enfermería con labores de información y una trabajadora social). Posteriormente, una vez se habían analizado, una vez se habían analizado los datos previamente recogidos mediante las entrevistas, se celebró el grupo de discusión, ya que uno de los objetivos que se perseguía con su realización era corroborar, contrastar y ampliar los temas que previamente hubieran resultado ambiguos, no suficientemente desarrollados o contradictorios, de manera que la combinación de los datos obtenidos mediante las dos técnicas pudiera aportar mayor fiabilidad a la investigación. También en el caso del grupo de discusión la muestra seleccionada presentaba un carácter heterogéneo y estaba compuesta por tres obstetras, dos matronas y una enfermera.

Tanto las entrevistas como el grupo de discusión se grabaron en formato audio y, a continuación, se transcribieron literalmente (verbatim) para facilitar su posterior análisis. ${ }^{3}$ Las preguntas planteadas en ambos casos seguían un guion variable, que dependía del grupo al que estas iban dirigidas, y estaba sujeto a la introducción de modificaciones, variaciones o digresiones

2. Nos referimos especialmente, aunque no exclusivamente, a los documentos suministrados por la Administración pública, así como por parte de las dos entidades encargadas de ofrecer servicios de interpretación en los hospitales y centros de salud de esta ciudad aragonesa.

3. Para el análisis de las transcripciones nos apoyamos en el programa de análisis de datos cualitativos NVivo10, lo cual nos permitió codificar y clasificar las respuestas obtenidas en los diferentes bloques temáticos que incluiríamos en el posterior informe de resultados de la investigación. 
propiciadas por las propias respuestas recibidas. Asimismo, se trataba de preguntas abiertas, de manera que los participantes pudieran expresar sus experiencias sin ceñirse a las perspectivas de la propia investigadora o a los resultados de investigaciones previas. Estaban divididas, además, en diferentes secciones, que se centraban en aspectos tales como la experiencia general en la asistencia a usuarias de origen extranjero, la interacción con las mismas o las actitudes sociales que estas demostraban. Una de las secciones destacadas, en la que nos basamos para presentar las opiniones de los profesionales sanitarios con respecto al tema que nos ocupa, abordaba las barreras lingüísticas que existían en la comunicación con las usuarias alófonas y las soluciones adoptadas por el hospital y su personal para tratar de mitigarlas o eliminarlas. ${ }^{4}$

Dividimos el análisis de los resultados en diferentes subapartados, los cuales se centran respectivamente en las estrategias implementadas en el contexto de estudio para reducir o eliminar las barreras lingüísticas que surgen en la comunicación con los pacientes alófonos, y en la opinión y la actitud de los profesionales sanitarios que han participado en nuestra investigación relativas a dichas barreras, a las soluciones adoptadas para superarlas y al rol del intérprete.

\section{Principales resultados del estudio}

En este apartado, como hemos señalado previamente, presentamos, en primer lugar, las principales estrategias que se han empleado en los servicios sanitarios de la ciudad objeto de análisis para tratar de superar las barreras lingüísticas que surgen en la comunicación con los pacientes que no dominan la lengua mayoritaria, e indicamos brevemente las principales ventajas e inconvenientes de cada una de ellas. A continuación, exponemos la opinión y la actitud de los profesionales sanitarios que han participado en nuestra investigación con respecto a las mencionadas barreras y las soluciones que estos suelen adoptar para superarlas. En un último subapartado incluimos la percepción de los profesionales sanitarios participantes en relación con el papel y las funciones del intérprete y los requisitos que este debe cumplir para desempeñar adecuadamente su labor.

4. En posteriores apartados se presentan las preguntas concretas planteadas a los profesionales participantes en las entrevistas y los grupos de discusión por lo que respecta a las cuestiones analizadas en el presente artículo. 
3.1. Estrategias empleadas en el hospital contexto de estudio para facilitar la comunicación con usuarias alófonas

En el contexto que nos ocupa se han utilizado en la última década diferentes estrategias de forma simultánea o alterna con el fin de mejorar la comunicación entre los profesionales sanitarios y las usuarias alófonas, entre las cuales se encuentran los servicios de interpretación prestados por diferentes figuras.

Por una parte, se ha empleado la interpretación presencial, llevada a cabo por intérpretes ad hoc, tanto familiares y amigos del propio paciente como miembros del Servicio Permanente de Interpretación gestionado por un centro social municipal dirigido a inmigrantes y minorías étnicas.

El recurso a familiares y amigos, el cual, por los datos obtenidos en la investigación, parece ser la solución más comúnmente adoptada en el hospital analizado, puede conllevar algunas ventajas, como por ejemplo, su disponibilidad a cualquier hora, el conocimiento previo por parte de dichos familiares y amigos de los problemas de salud del paciente, o la tranquilidad que puede transmitir a este último su presencia. Sin embargo, el desempeño de labores de interpretación por parte de estas personas implica también considerables riesgos. Así, por ejemplo, pueden ofrecer su opinión o su propia versión de los hechos en lugar de ceñirse a los mensajes transmitidos por los interlocutores, pueden decidir omitir determinados datos para proteger al paciente, o pueden sentirse molestos o violentos (tanto ellos mismos como el propio paciente) al tratar determinadas cuestiones de carácter más privado o delicado. Asimismo, la intimidad del paciente queda ulteriormente comprometida cuando la interpretación es llevada a cabo no por algún miembro de su familia o círculo de amistades, sino por otras personas ajenas, como pueden ser los acompañantes de otros pacientes.

Por lo que respecta al mencionado Servicio de Interpretación, que se empezó a prestar en 1999 fruto de un convenio entre el Ayuntamiento y el Colegio de Abogados de la ciudad contexto de estudio y se suprimió en marzo de 2012, presentaba una disponibilidad limitada (en particular, de lunes a viernes, de las 9:00 a las 21:00 horas). Además, a los intérpretes inscritos en su registro, los cuales eran principalmente inmigrantes, se les exigía únicamente que se encontraran en situación regular. Por el contrario, no se les solicitaba ningún tipo de certificado o acreditación que demostrase sus conocimientos lingüísticos de las lenguas de trabajo ni formación en interpretación. Asimismo, estos intérpretes no se regían por ningún código deontológico 
ni se efectuaba ninguna valoración posterior de la calidad de los servicios prestados. ${ }^{5}$

Son considerables las posibilidades de que, al emplear estas dos soluciones descritas hasta el momento, la comunicación entre los profesionales sanitarios y los pacientes alófonos no sea efectiva, principalmente por la falta de preparación y formación previas de las personas que actúan como intérpretes ad hoc. De hecho, aparte de sus carencias por lo que respecta al conocimiento de las lenguas implicadas y de las técnicas básicas de interpretación, estos intérpretes probablemente no están preparados para afrontar las dificultades propias del desempeño de esta profesión, especialmente las de carácter psicológico, y desconocen las funciones y principios inherentes al papel que deben ejercer (Wadensjö 1998: 52).

En consecuencia, es bastante elevado el riesgo de que estos intérpretes ad hoc cometan los cinco errores básicos identificados por Vásquez y Javier (1991, citados en Hale 2010: 48). Así, podrán producir errores de omisión (cuando el intérprete suprime total o parcialmente el mensaje transmitido por un interlocutor), de ampliación (o inclusión de información no expresada por los interlocutores), de condensación (o tendencia a simplificar o sintetizar el mensaje de un interlocutor), de sustitución (o tendencia a remplazar conceptos), o de intercambio de roles (cuando el intérprete asume el papel de uno de los interlocutores y es él mismo quien realiza preguntas o da respuestas).

La falta de formación puede conducir también al incumplimiento de determinados principios contenidos en la mayoría de los códigos deontológicos de asociaciones de intérpretes médicos ${ }^{6}$ tales como los principios de fidelidad al mensaje (al omitir, añadir o interpretar personalmente determinada información), de imparcialidad (por ejemplo, debido a la dificultad de distanciarse emocionalmente, por pertenecer a la misma familia o comunidad del paciente), o de confidencialidad.

El desempeño de la interpretación en ámbito sanitario por personas no debidamente formadas supone además un gran impacto emocional para las

5. La información presentada en este párrafo ha sido extraída de una entrevista realizada a la responsable de gestionar el servicio de interpretación en el mencionado centro municipal y de la documentación oficial que esta misma persona nos entregó.

6. Al respecto, véanse, por ejemplo, los siguientes documentos: California Standards for Healthcare Interpreters: Ethical Principles, Protocols and Guidance on Roles \& Intervention (CHIA, California Healthcare Interpreters Association, EEUU, 2002); IMIA Standards of Practice (International Medical Interpreters Association, EEUU, 2007); National Standard Guide for Community Interpreting Services (Healthcare Interpretation Network, Canadá, 2007); National Standards of Practice for Interpreters in Healthcare, (National Council on Interpreting in Healthcare, EEUU, 2005). 
mismas, especialmente en el caso de los menores, por su cambio de roles en el contexto familiar y por recibir información de carácter demasiado grave para su grado de madurez.

En última instancia, por las carencias descritas, el recurso a la interpretación ad hoc puede conllevar consecuencias negativas relacionadas con la propia asistencia sanitaria prestada, como "reduced trust in physicians, lower patient satisfaction [...], misdiagnosis, inadequate or inaccurate treatment, and reduced quality of care" (Jacobs et al. 2001: 469, citados en Angelelli 2008: 23).

Aparte de las dos soluciones ad hoc mencionadas, desde junio de 2009 se implantó en los centros de salud y hospitales de la ciudad contexto de estudio un servicio de interpretación telefónica gestionado por una empresa privada externa a la Administración pública, el cual ha seguido ofreciéndose en periodos discontinuos hasta el momento presente. Con respecto a la disponibilidad, en el caso de los centros de salud y hospitales de la ciudad contexto de estudio, el servicio se oferta las veinticuatro horas durante todos los días del año. Por consiguiente, a diferencia del servicio de interpretación presencial previamente analizado, este no presenta ninguna restricción en cuanto al horario o calendario de disponibilidad. Por lo que se refiere a las condiciones técnicas del servicio, la empresa distribuye unos terminales con doble auricular a los centros donde este se presta, de manera que es posible establecer una conversación tripartita (en la cual, en todo momento, ambos interlocutores pueden escuchar la conversación y la intervención del intérprete), y se evita la incomodidad de tener que ir pasando el auricular de uno a otro interlocutor. ${ }^{7}$

Las principales ventajas de este servicio son, por una parte, el empleo de intérpretes profesionales (los cuales cuentan con formación reglada en traducción e interpretación o, en su defecto, deben superar una prueba que determina su capacidad para interpretar telefónicamente) y, por otra, y en comparación con la interpretación presencial, la reducción de costes y la inmediatez.

Por lo que respecta a las desventajas, la distancia física entre el intérprete y los interlocutores, con la consiguiente ausencia de un marco de referencia compartido y de visión de los elementos no verbales, pueden conducir a omisiones o malentendidos. Así, como sostiene Wadensjö (1999: 254), el intérprete telefónico, en comparación con el presencial, no puede captar la

7. La información presentada en relación con el servicio de interpretación telefónica, por un lado, ha sido extraída de los pliegos oficiales de prescripciones técnicas y, por otro, se corresponde con los datos proporcionados por la propia empresa prestataria. 
información transmitida por los interlocutores a través de los gestos, la postura, la mímica y otros elementos del lenguaje no verbal, la cual juega un papel esencial en el acto comunicativo y, por tanto, es de gran relevancia para el ejercicio de la interpretación.

Cabe señalar, de todos modos, que la ausencia física del profesional responsable de llevar a cabo la interpretación telefónica puede constituir asimismo un factor positivo, puesto que garantiza que exista mayor confidencialidad en situaciones comprometidas (como podrían ser las consultas médicas en las que se realizan exploraciones) y, al mismo tiempo, puede mitigar la preferencia de un intérprete de un determinado sexo por parte de algunos usuarios (Mikkelson 2003: 260).

Para finalizar, en el contexto estudiado, la principal desventaja que presenta este servicio es, sin duda, y como veremos más en detalle en los próximos subapartados, su desconocimiento y su infrautilización, causada en parte por la actitud que presentan los profesionales sanitarios con respecto a las barreras lingüísticas que surgen en la comunicación con pacientes alófonos y por el concepto que estos profesionales tienen del rol del intérprete y de los requisitos necesarios para realizar una adecuada interpretación.

\subsection{La opinión y la actitud de los profesionales sanitarios}

En este subapartado presentamos, como su título indica, la opinión y la actitud manifestada por los profesionales sanitarios del hospital analizado con respecto a las barreras lingüísticas que surgen en la comunicación con las usuarias alófonas y las estrategias para superar dichas barreras. Con el fin de ilustrar nuestros hallazgos, incluimos fragmentos de las entrevistas y el grupo de discusión, ${ }^{8}$ de manera que damos voz a los propios participantes en nuestra investigación.

8. Para garantizar el anonimato de los participantes en el estudio se les identifica, en el caso de las entrevistas, con el perfil profesional y un número que se corresponde con el orden cronológico en el que estas se realizaron. Por lo que respecta al grupo de discusión, se introduce la etiqueta identificativa del participante con la sigla GD, la cual se acompaña del perfil profesional y de un número que señala el orden en el que se tomaron sus datos personales. Cabe además indicar que la participación presentaba un carácter voluntario y que todos los sujetos firmaron un consentimiento informado mediante el cual manifestaban su disponibilidad para colaborar en la investigación. 
3.2.1. ¿Qué opinan los profesionales sanitarios sobre las barreras lingüísticas?

Con respecto a la opinión de los profesionales sanitarios sobre los obstáculos a los que se enfrentan a la hora de relacionarse con las usuarias de origen extranjero, cabe destacar que, desde el principio de las entrevistas y el grupo de discusión, los profesionales sanitarios señalan las barreras lingüísticas como la causa fundamental de las dificultades que se plantean en la asistencia a dichas usuarias. De hecho, son las primeras barreras mencionadas ante una de las primeras preguntas realizadas al comenzar las entrevistas, la cual decía exactamente: "En caso de considerar los problemas con las usuarias de origen extranjero diferentes o mayores a los que encuentra con las usuarias españolas, ¿cuáles cree que son las causas principales de dichos problemas?". Asimismo, se trata de la principal causa señalada por los participantes en el grupo de discusión al preguntarles "¿Cuáles creen que son las posibles causas de las dificultades para tratar a usuarias de origen extranjero?"

Sin embargo, a pesar de la relevancia concedida a estas barreras, ante la pregunta concreta "¿Qué tipo de problemas lingüísticos suelen presentar las usuarias de origen extranjero?" en las entrevistas, o la pregunta "¿Existen barreras lingüísticas al comunicarse con las usuarias de origen extranjero?" en el grupo de discusión, los participantes en el estudio presentan dos actitudes discordantes al respecto.

Por una parte, algunos profesionales sanitarios restan importancia a los problemas comunicativos causados por la ausencia de una lengua común. Consideran que actualmente son muy pocas las usuarias que no hablan nada de español y que siempre, de uno u otro modo, han conseguido comunicarse.

- De todos modos, la verdad es que encontrarte con una paciente que no sepa nada, nada, nada de español es difícil. Todas saben alguna palabra. [Anestesista 5]

- Vamos, para simplemente entenderte, yo creo que te puedes entender con cuatro palabras, que son las que te sirven para saber si hay alguna patología o si todo entra dentro de la normalidad. [Matrona 2]

- Yo diría que siempre de algún modo esa barrera la hemos conseguido superar. Y, fijate, que hemos tenido gente de todas partes, y nunca he tenido problema para comunicarme. Yo creo que la comunicación depende de cada uno, si uno quiere comunicarse, por un medio o por otro lo conseguirá. [Obstetra 1] 
Por otra parte, están aquellos profesionales sanitarios (una gran mayoría) que consideran que las barreras lingüísticas suponen un obstáculo importante, incluso el mayor al que deben hacer frente en la asistencia a usuarias inmigrantes, ya que opinan que el hecho de no conocer el español impide que estas entiendan las explicaciones que se les ofrecen o que ellas mismas se puedan expresar.

- Los problemas con las usuarias inmigrantes son mayores. Sobre todo con aquellas que no entienden español y no hablan. Pero sobre todo con las que no entienden, porque no les puedes explicar nada de lo que les vas a hacer. Nosotros necesitamos que ellas colaboren y nos den una serie de datos y poder explicarles un poco en qué consiste lo que les vamos a hacer. Y si no hablan español, esto es imposible. [Anestesista 2]

- El desconocimiento de la lengua creo que es, al menos para mí, una cosa fundamental. El prestar una atención correcta a una persona que no sabes con qué te puedes enfrentar, esto es como irte... yo qué sé... como irte a la sabana y decir Vamos a ver si tengo a los lobos ahí esperándome. [Anestesista 3]

La imposibilidad de comunicación entre profesionales y usuarias alófonas puede desembocar, de acuerdo con algunos profesionales, en graves consecuencias médicas o incluso legales, especialmente referidas en el contexto que nos ocupa al suministro de la anestesia epidural.

- Para mí la mayor barrera es la lingüística. [... es es que en una anestesia epidural, con el volumen de anestésico que yo estoy poniendo, si lo pongo en el sitio erróneo, es que te cargas a una persona, es que te la cargas, así de sencillo, ¿vale? Entonces, tienes que tener unos métodos para intentar comprobar que tú lo estás haciendo bien, y los métodos son no solo farmacológicos, sino que yo tengo que preguntar a la gente cómo se siente, qué es lo que nota, si se le duermen las piernas, si está todo bien... Y si yo no tengo una comunicación que sea fluida, pues... [Anestesista 3]

- En teoría según la legislación que existe en nuestro país, la paciente tiene que entenderlo y tenemos además que contarle todas las opciones que hay, cómo funcionan y lo que puede pasar con cada una de ellas. Y ella es libre de decir si quiere, cuándo quiere... pero con las que no hablan tu idioma a veces esto es muy difícil hacerlo. [Anestesista 4] 
- A ver, hay un problema legal. Realmente, en otros hospitales donde he trabajado, si tú no te entiendes con el paciente, no deberías hacer ninguna técnica .... vital, o sea, perdón, no vital. Por ejemplo, la epidural es no vital, puedes parir con o sin epidural. Si nos ponemos estrictos, si tú no puedes mantener una conversación normal con la paciente, no deberías aplicarle técnicas que ella puede no entender. [Anestesista 5]

Para finalizar este subapartado, cabe mencionar que, al hablar de las barreras lingüísticas, en ocasiones, los profesionales sanitarios realizan una diferenciación entre las usuarias dependiendo de su país de origen y, como puede observarse en los fragmentos de entrevistas que incluimos a continuación, opinan que la comunicación suele ser más fluida con las usuarias latinoamericanas y las procedentes de Europa del este, mientras que las usuarias de Asia, de África Subsahariana y del Magreb suelen presentar mayores problemas de comunicación. Estos datos podrían servir como un primer indicador para, posteriormente, llevar a cabo una investigación detallada de las necesidades específicas del contexto de estudio y poder ofrecer una respuesta adecuada a las mismas.

- Y también según... digamos la... no sé cómo decirlo, según la raza, ¿sabes?, o el origen, pues es mucho más difícil la comunicación, por ejemplo a la hora de preguntar por las alergias y tal. Los chinos sí que siempre traen un acompañante, normalmente nunca están solas. Las rumanas y tal sí que se desenvuelven muy bien en español. Así que normalmente este tipo de problemas pues son más con árabes y con gente de color. [Enfermera 1]

- A ver, por ejemplo, los del este sí que la mayoría son muy espabilados y saben más que... más que nosotros. Con los sudamericanos evidentemente no hay barrera lingüística. Ahí, estupendamente. Y las marroquíes, las argelinas... vienen algunas que no entienden absolutamente nada, sobre todo las que han pasado allí el embarazo y vienen a parir aquí. [Matrona 5]

- Barreras lingüísticas, con las rumanas, por ejemplo, no, porque las rumanas entienden bastante, se desenvuelven muy bien. El problema es sobre todo con las chinas y las marroquíes, principalmente. Las marroquíes te das cuenta que igual llevan aquí diez años y no hablan (español), igual que algunas chinas. [Obstetra 3] 
3.2.2. ¿Qué estrategias emplean los profesionales sanitarios para reducir o eliminar las barreras lingüísticas?

Tanto en las entrevistas como en el grupo de discusión se trató de analizar las estrategias que los profesionales sanitarios utilizan para tratar de superar las barreras lingüísticas. En particular, en las entrevistas se formularon las siguientes preguntas:

- ¿Cómo suelen resolver los problemas lingüísticos?

- ¿Cuenta el centro con intérpretes? ¿Son estos oficiales, voluntarios...? $¿ \mathrm{O}$ son los propios pacientes los que traen a alguien que les traduzca?

- ¿Hay algún servicio dependiente de los organismos oficiales al que puedan recurrir para solicitar intérpretes presenciales o vía telefónica? En caso afirmativo, ¿emplean dicho servicio?

Y, en el caso del grupo de discusión, las preguntas realizadas fueron:

- ¿Cómo se eliminan las barreras lingüísticas?

- ¿Qué resultados tienen los métodos utilizados?

- ¿Se les ocurren otras propuestas para eliminar estas barreras?

De acuerdo con los datos proporcionados por los profesionales sanitarios participantes en la investigación, el recurso a familiares o amigos de las usuarias alófonas para que realicen labores de interpretación es el método más empleado en el hospital estudiado para comunicarse con dichas usuarias.

- Son los pacientes los que generalmente traen a alguien que les traduzca. Suele ser alguien de la comunidad o un familiar que lleva más años en el país y un poco controla el idioma. [Anestesista 1]

- Suelen venir con familiares, amigos... que saben más o menos hablar español, y entonces con la gran mayoría no tenemos ningún problema en que pasen, y les expliquen las cosas y estén con ellas. [Matrona 4]

Por la información transmitida por los participantes en el estudio, descubrimos que estos intérpretes ad hoc no siempre son familiares directos o amigos de las usuarias, sino que en ocasiones se ha recurrido a otras usuarias ingresadas en el hospital y a los acompañantes de las mismas, a personas desconocidas con las que se establece comunicación telefónica, o incluso a niños.

- Sí, sí hemos utilizado a menores para hacer de traductor. Y, a veces, incluso he utilizado el teléfono móvil para hablar con familiares y 
explicarles a ellos las cosas. Pues el sobrino sabe español y chino, y traduce a su tío español y chino. [Anestesista 5]

- Según qué personas hay, las uso de intérpretes yo. Les digo ¿Tú eres rumana y hablas rumano y español? Pues, vente conmigo que me vas a ayudar a preguntarle a esta paciente. Vamos, hago como conjuntos. Con los árabes, también. Y ellos colaboran muy bien. [Enfermera 5]

- Yo, cuando ha hecho mucha falta, incluso he buscado, por ejemplo... necesito una persona que habla un dialecto africano, pues me he recorrido las plantas buscando a algún negro que sea capaz de traducir. [Profesional socio-sanitario 1]

- Se recurre muchas veces a parientes, amigos, o incluso compatriotas que se encuentran en el hospital. ¿Y niños? ¿Para interpretar a sus padres? Sí, los hemos utilizado muchas veces. Los críos son más hábiles aprendiendo los idiomas y hay fundamentalmente mujeres que no hay forma de que lo aprendan y sus hijos les traducen. [Profesional socio-sanitario 1]

Son numerosos los profesionales sanitarios que ven ventajas en el empleo de familiares y amigos para que realicen labores de interpretación, como su amplia disponibilidad horaria o la cercanía de estos con la usuaria. Estos factores, junto con otros (como el ahorro de costes o la disponibilidad de personal sanitario bilingüe) podrían ser determinantes, como se indica en estudios previos (Diamond et al. 2009: 258-259; Bischoff \& Hudelson 2010a: 2842; Bischoff \& Hudelson 2010b: 17), a la hora de recurrir a intérpretes ad hoc en lugar de a intérpretes profesionales.

- [...] con las que no hablan nada, nada, nada, desde luego el acompañante sirve de traductor. Y la experiencia con el acompañante que hace de traductor es útil, yo creo que sirve, sí. [Anestesista 5]

- Pues, a ver, lo que yo veo es que si la persona (el acompañante que hace de intérprete) está aquí, siempre en algún momento, cuando tengas una duda, pues siempre puedes recurrir a ella. Y, aunque la comunicación no sea muy fluida, siempre está ahí, ¿sabes? [Enfermera 1]

- Cuando el marido o un familiar hacen de intérprete, generalmente, sí que se solucionan los problemas de comunicación. Porque él les explica y así... [Matrona 7]

- Muchas veces sí que hemos utilizado como intérprete a algún familiar o amigo que hablara español y la lengua de la paciente. Si vemos que entre ellos hay una buena relación, casi es preferible, porque si es un 
familiar íntimo, que a la paciente no le importe que se entere de cosas, entonces, pues casi es mejor... [Obstetra 1]

Asimismo, algunos parecen considerar incluso como una obligación de las propias usuarias el acudir al hospital acompañadas por alguien que les ayude a comunicarse en caso de que estas desconozcan el español.

- En general (las usuarias) no vienen con alguien que les traduzca, no vienen preparadas, lo que pasa es que aquí se les dice que, hombre, sería mejor, ¿no? Pero yo no creo que tengan la concepción de que tengan que buscar a alguien que les traduzca y eso, simplemente vienen, y vienen, sin preocuparse de si les entienden o no. [Anestesista 2]

- Bueno, claro, hay muchas señoras, muchas mujeres, que cuando llegan aquí no tienen ni idea de nuestro idioma, y entonces les aconsejamos siempre que se busquen a alguien que les haga de intérprete. [Obstetra 1]

- Yo también me hice un cartel que pone Por favor, vengan acompañados de alguien que hable nuestra lengua, porque acudían a las consultas sin decir ni media palabra, ni acompañante, ni habiéndolo solicitado previamente, con lo cual no daba tiempo de solicitarlo y tal... [Profesional socio-sanitario 1]

Sin embargo, otros profesionales sanitarios son conscientes de los problemas que puede ocasionar la falta de preparación y formación de los familiares y amigos de la usuaria para ejercer como intérpretes. Al respecto, algunos señalan la falta de conocimientos lingüísticos generales, o el desconocimiento de la terminología y los conceptos básicos propios del ámbito sanitario.

- Normalmente te traduce el marido, [...] que tampoco es gente que tenga unos conocimientos... o sea, que tiene que ser todo como muy casero, vamos, tenemos que explicárselo a él para que se lo pueda explicar. [Anestesista 2]

- Y es que la comunicación no es la misma. Porque tú estás con una persona que le está contando bien, se supone, lo que tú quieres decir, pero es que hay muchas ocasiones en las que, hablando con esa persona, te das cuenta de que tampoco te entiende al cien por cien lo que tú le estás diciendo. Entonces dices, muchas veces dices ¿Le estará diciendo lo que yo quiero que le diga? [Anestesista 3]

- [...] porque si el intérprete es un familiar, a lo mejor, según qué palabras ya más especializadas, pues a lo mejor, no sabe explicárselas o 
decírselas en su idioma. Me refiero a términos médicos y cosas de esas... [Obstetra 2]

Además, diversos profesionales sanitarios entrevistados muestran preocupación con respecto al posible incumplimiento de los principios de fidelidad e imparcialidad, y presentan dudas acerca de si existen omisiones, ampliaciones, condensaciones o intercambio de roles por parte de estos intérpretes ad hoc, errores que, como se demuestra en estudios anteriores (como, por ejemplo, en Flores et al 2012), suelen cometer los intérpretes sin formación.

- Muchas veces tienes que insistir No, pero pregúntale si... Porque también a veces pasa que, si quien traduce es la pareja o el acompañante, que se supone que es el marido, te contesta él sin hablar con ella. [Anestesista 2]

- Si un familiar o conocido hace de intérprete, pues ayudar sí que te ayuda, pero claro, siempre te queda la duda de... de lo que realmente le está transmitiendo, si realmente le está diciendo toda la información que tú quieres o cómo la está transmitiendo. [Enfermera 3]

- Cuando el marido o un familiar hacen de intérpretes a veces sí que puedes tener el problema de que no sabes si lo que le estás diciendo se lo está traduciendo tal cual o le está cambiando la versión. [Matrona 2]

Por otra parte, algunos profesionales sanitarios desconfían de que, al emplear a familiares o amigos como intérpretes, se mantenga la confidencialidad o la intimidad necesarias en este contexto, tal y como queda demostrado en estudios previos (como, por ejemplo, Leanza 2007: 12 o Bischoff $\&$ Hudelson 2010a: 2839).

- $[\ldots]$ o te los pasan por teléfono y te explican que es el primo de... que realmente te preguntas hasta qué punto se respeta la protección de datos o de la intimidad del paciente. [Enfermera 1]

- [... también depende un poco de la relación que tenga esa mujer con el intérprete, porque estás en un momento muy importante, ¿no?, muy significativo y muy íntimo. Y, a lo mejor, estar con una persona que es la primera vez que la ves o que es algún amigo de la familia, pero que... Y, entonces, a lo mejor hay cosas que la mujer no expresa porque no se las va a decir a él para que te las diga... O puede haber situaciones que te resultan un poco... un poco violentas. Vamos, que depende de la relación que tengan. [Matrona 4] 
Estas mismas preocupaciones por la calidad de la interpretación cuando se recurre a familiares y amigos quedan reflejadas en el grupo de discusión, tal y como puede observarse en el fragmento que se incluye a continuación.

- Y, normalmente, les pides si pueden entrar con alguien que entienda o que hable español más que ellas. Lo que pasa es que a veces, yo qué sé... [GD - Matrona 2]

- (Les pregunto si, cuando han usado como intérpretes a parientes o amigos, la comunicación ha resultado eficaz.)

- A veces te encuentras con que el intérprete sabe menos que ellas. [GD - Obstetra 2]

- Exacto, que el intérprete sabe menos, o no estás realmente convencida de todo lo que se está traduciendo, ¿no? O que no sabes lo que le está llegando, porque, claro, como tú no tienes ni idea, ni tienes mucha confianza..." [GD - Matrona 2]

- A veces, es verdad, que no te quedas del todo convencido. Y, luego, también depende de qué grado de familiaridad o de relación tiene con la paciente. Porque si es la madre y, como pasa a veces con las españolas, que es la madre la que sabe y la que te contesta cuándo tiene su hija la regla y no sé cuántos, pues... Te la lía, esa sí que te la lía, porque ahí sí que intuyes que la mujer se ve apurada y no está entendiendo qué..." [GD - Obstetra 2]

Por lo que se refiere a los servicios de interpretación proporcionados por entidades externas de los que hemos hablado en el subapartado anterior, la primera cuestión que podríamos destacar es el gran desconocimiento y la infrautilización de los mismos.

- No lo sé. La verdad es que los intérpretes que hay no sé de quién dependen, si es un servicio oficial o si es algo voluntario. El centro, propiamente, yo creo que no cuenta con intérpretes. Hay intérpretes que creo que si se les llama, vienen. Pero que trabajen aquí y eso, no. [Anestesista 2]

- No, no, el centro no cuenta con intérpretes. [Anestesista 3]

- Sé que hay forma de resolver los problemas lingüísticos, parece ser que con teléfonos y tal, pero yo no lo he utilizado, no, no lo he utilizado. [Anestesista 5]

- No, no conozco ese servicio de interpretación telefónica del que me hablas y, por supuesto, no lo he empleado nunca. [...] Pero traductores presenciales, creo que sí que hay. [Matrona 2] 
- Hasta ahora existía un servicio de... guía telefónica, que podías hablar, les llamabas y decías Oye, éste es ruso, o es senegalés... Pero creo que llevamos ya varios meses que ya no existe ese móvil con el que te ponías en contacto con los traductores. [Profesional socio-sanitario 3]

Algunos profesionales sanitarios no ven la necesidad de emplear dichos servicios y afirman que consiguen comunicarse con las usuarias alófonas adoptando otras soluciones, principalmente el recurso a familiares y amigos mencionado previamente. No tienen en cuenta que, como demuestran estudios previos (Flores 2005; Flores et al. 2012), son mayores las probabilidades de que estos intérpretes no formados cometan errores con graves consecuencias. Asimismo, parecen restar importancia al hecho de que, como se demuestra en diferentes estudios (como, por ejemplo, Diamond et al. 2009: 256 o Nápoles et al. 2010: 302) el uso de intérpretes profesionales garantiza una comunicación más efectiva con los pacientes alófonos y, en consecuencia, una mayor satisfacción de dichos pacientes, una reducción de las desigualdades de acceso a los servicios de salud y una mejora en la asistencia sanitaria prestada.

- Pues no sabía que había un servicio de interpretación telefónica, pero si no lo usan, será porque siempre hay alguien de la familia o del entorno de la paciente que les puede ayudar. [Anestesista 2]

- Claro, es que yo creo que recurres a ello cuando ya... cuando la comunicación es nula, pero si te vas apañando con lo poco que puedan saber ellas, con lo que te cuenta el marido, o con lo que sea, pues por eso igual no se utiliza... [Matrona 3]

- Sé que existe un servicio de interpretación telefónica, pero no lo he usado nunca. Porque como siempre ha habido alguien que ha solucionado el problema de la comunicación, no ha hecho falta. [...] Pero aquí no contamos con ningún intérprete oficial. Lo que sí que se hace, como te decía antes, es recurrir a algún familiar o a un amigo que hable español. [Matrona 6]

Concretamente, con respecto al servicio de interpretación presencial que, hasta marzo de 2012, gestionaba un centro municipal, algunos profesionales sanitarios apuntan como desventaja la limitación horaria y el hecho de que se tuviera que solicitar con antelación y no se pudiera contar con el intérprete de manera inmediata, hecho que puede haber fomentado el recurso a familiares y amigos, como queda demostrado en otros estudios previos citados con anterioridad (Diamond et al. 2009; Bischoff \& Hudelson 2010b; Flores et al. 2012). 
- Pues creo que sí que hay intérpretes, pero, claro, no están las veinticuatro horas. [Matrona 4]

- El problema de los intérpretes profesionales es su horario limitado. Por ejemplo, en urgencias, cuando los necesitas, a veces no coincide con el horario del intérprete. [Obstetra 3]

- Antes, cuando trabajábamos con el centro municipal, se solicitaba el servicio de traducción previamente, tenían que avisar de que iban a venir, y entonces lo pedíamos. Pero, inmediatamente, era imposible, porque ellos tenían que contactar con el traductor y que viniera. [Profesional socio-sanitario 1]

Y en cuanto al servicio de interpretación telefónica, son muy pocos los profesionales sanitarios participantes en nuestro estudio que lo han empleado. Aducen esta infrautilización al desconocimiento del funcionamiento del servicio (a pesar de que nos consta que en el hospital analizado se han realizado campañas informativas al respecto) $\mathrm{u}$ otros motivos, entre los cuales se destaca que se trata de un recurso complicado, incómodo, con el que se pierde cercanía con la usuaria o que requiere que empleen más tiempo del habitual, siendo esto algo que escasea en su rutina laboral.

- En el hospital no contamos con ningún traductor, y el servicio de traducciones telefónicas ni nos dicen muy bien cómo funciona, ni sabes a qué horas está disponible, ni dónde está.... Vamos, que a mí nadie me ha dicho dónde tengo que llamar, ni cómo tengo que hacerlo, ni a qué hora, ni a qué empresa, ni si esas conversaciones quedan grabadas o no quedan grabadas, no sé si lo que se está diciendo... sí, sé que me tengo que fiar porque es un traductor, pero lo que no sé es cómo funciona eso... [Anestesista 4]

- Yo sé que había por ahí un papel circulando que decía que se puede llamar a un intérprete que te ayuda por teléfono. Pero, chica, a mí eso no me ha llegado, ni sé cómo funciona, ni... Yo sé que las compañeras de admisión lo leyeron en la revista, que lo iban a poner y tal, pero ni siquiera sé si finalmente lo han puesto o... [Profesional socio-sanitario 2]

- Con ese servicio se pierde mucho tiempo, si estás al teléfono yo creo que en consulta lo que son 15 minutos te cuesta una hora y pico, y eso es inviable... Así que el acompañante hace de traductor y ya está. [Anestesista 4]

- Sé que hay un servicio de interpretación telefónica, pero yo nunca lo he utilizado. A veces... yo creo que es complicado y, además, 
hacerlo... no sé, a través del teléfono yo creo que también se pierde un poquito de... de intimidad, de cercanía con la mujer, con lo que te está diciendo a través del teléfono la otra persona... [Enfermera 3]

- Y si no te entiendes nada, nada, nada, pues hay un teléfono en urgencias con el que te puedes poner en contacto con algún traductor. Pero, claro, no es tan fácil tampoco a veces... Digamos que lo del teléfono para ponerse en contacto con un traductor yo lo uso ya como último recurso. Yo creo que es un poco por el tiempo. Pensamos que con el teléfono vamos a perder más tiempo o... Como que te intentas apañar, y no sé si no lo usamos por tiempo o por intentar solucionar tú mismo la cosa. [Obstetra 2]

Tanto el escaso empleo del servicio de interpretación telefónica como el desconocimiento del mismo y de su funcionamiento quedan constatados en el grupo de discusión, de cuyos participantes solo uno lo ha utilizado, tal y como puede observarse en el fragmento que incluimos a continuación.

- Si no, recurrimos al traductor del hospital. [GD - Obstetra 3]

- Ya, pero depende de cómo te pille... Porque yo reconozco que si me pilla en el ambulatorio, con un montón de mujeres esperando y tal, pues... [GD - Obstetra 1]

- (Le pregunto a GD - Obstetra 3 si, cuando ha hablado del traductor del hospital, se refería a un servicio de traducción/interpretación en concreto.)

- Me refiero al de urgencias. Ese es el que yo he utilizado. El servicio de interpretación por teléfono. [GD - Obstetra 3]

- (Le solicito, visto que tiene experiencia empleando el servicio de interpretación telefónica, cómo le ha resultado, si lo ve complicado o sencillo.)

- No, bien. Bueno, yo lo he usado dos o tres veces y... siempre me ha ido bien. Lo he usado siempre por el día, ¿eh? (Le comento que el servicio está disponible veinticuatro horas al día.) Ah, ¿está veinticuatro horas? No lo sabía... Pues, bueno, por el día, bien, a mí me ha funcionado bien. Ahora sí, hay que tener tiempo para usarlo, porque, por ejemplo, hay que poner muchos códigos y... ${ }^{9}$ Pero sé que se usa

9. Cabe señalar que el número de códigos que se deben introducir antes de contactar con el intérprete telefónico es uno de los aspectos que, de acuerdo con la responsable del Gobierno de Aragón encargada de gestionar este servicio, se mejorarán en el próximo periodo de adjudicación, pues son conscientes de la pérdida de tiempo que esto implica. 
poco, e incluso hay profesionales que no saben ni que existe, o que no saben cómo se usa... [GD - Obstetra 3]

- Por ejemplo, yo no sé cómo se usa. [GD - Obstetra 1]

- Pregunto al resto de participantes si algún otro ha usado este servicio y todos niegan, verbalmente o mediante un movimiento de cabeza, haberlo empleado.)

- No, normalmente se recurre a algún pariente o amigo de la paciente y... [GD - Obstetra 2]

- (Les pregunto qué inconvenientes ven a la interpretación telefónica y por qué creen que no se usa.)

- Hombre, es que es muy impersonal, ¿no? Estás ahí a través del teléfono... Vamos, a mí no me gusta. [...] la verdad, es que a lo que más recurrimos es al intérprete que es pariente y amigo. Porque, a mí, cuando me vienen con un teléfono y le tienes que contar a un tercero al que no estás viendo... [GD - Obstetra 2]

El hecho de que, aun existiendo servicios de interpretación profesional, no se recurra a los mismos, demuestra que no basta con ofrecer la posibilidad de recurrir a los mismos, sino que es necesaria una mayor profesionalización de esta figura y un mayor reconocimiento de la labor que desempeña por parte de los profesionales sanitarios y de las instituciones para las que estos trabajan (Bischoff \& Hudelson 2010b: 19; Brisett et al. 2013: 139).

\subsection{3. ¿Qué rol y qué requisitos atribuyen los profesionales sanitarios al intérprete?}

Por lo que se refiere a la opinión de los profesionales sanitarios sobre el rol y las funciones del intérprete, se formularon las siguientes preguntas en las entrevistas: "¿Cómo son en general las experiencias con una tercera persona haciendo de intérprete?" y "Por su experiencia, icree que cualquier persona con conocimientos lingüísticos de ambas lenguas puede ejercer de intérprete?".

Al respecto de esta cuestión, en primer lugar, cabe destacar el desconocimiento o confusión por parte de algunos con respecto a la terminología adecuada para referirse a los intérpretes y a la labor ejercida por los mismos, tal y como ha quedado reflejado en determinados fragmentos de las entrevistas y del grupo de discusión incluidos anteriormente, en los que los participantes empleaban indistintamente los vocablos "traductor", "traductor telefónico", "(servicio de) traducción telefónica", o incluso "guía telefónica". 
En cuanto al papel desempeñado por los intérpretes, la mayoría de los profesionales sanitarios opinan que su única labor consiste en traducir literalmente lo que dicen los interlocutores. De ello podemos deducir que ven al intérprete como un agente externo al acto de comunicación que se limita a reproducir automáticamente el mensaje en una y otra lengua. Esta misma percepción del intérprete como si se tratase de una translation machine ha sido señalada en estudios anteriores, como en el de Leanza (2005: 177), lo que denota que no se tiene en cuenta que "reproducing or rendering speech actions of others is a fairly creative activity" (Wadensjö 1998: 69).

No hay que interpretar, porque hacer de intérprete no significa interpretar lo que el otro quiere decir, sino que si el otro dice Pipí, sí, el intérprete dice $\mathrm{Ha}$ dicho: Pipí, sí. Como un notario. [Obstetra 1]

Además, algunos no observan grandes diferencias entre el empleo de familiares y amigos como intérpretes ad hoc y el recurso a intérpretes profesionales, y simplemente señalan que el recurso a la interpretación profesional puede ofrecerles mayores garantías sobre la fidelidad del mensaje transmitido, la cual, como se observará en uno de los fragmentos incluidos a continuación, en ocasiones se entiende como "transcripción literal".

No mencionan, sin embargo, otros de los principios por los que se debe regir un intérprete profesional (como puede ser la imparcialidad o la confidencialidad), ni la formación que este habrá recibido para desempeñar su labor, hechos que constituyen factores diferenciadores entre los intérpretes profesionales y los naturales (Flores et al. 2012: 548-550).

- Y, luego, también pasa que con el intérprete (profesional) sabes que se lo va a traducir todo tal cual, y el familiar también se lo puede contar un poco como él quiera. Eso lo he pensado muchas veces, sí. [Obstetra 2]

- No veo diferencias entre recurrir a un intérprete profesional o a una persona que simplemente conoce las dos lenguas. No, porque vamos, normalmente la gente pone interés en traducir. Lo único es que con uno profesional te queda la tranquilidad de que está haciendo la trascripción literal de lo que tú estás diciendo, que no interpreta ni pone nada de su cosecha. Es la única diferencia, porque con la otra forma te queda la duda de... [Profesional socio-sanitario 1]

Otra diferencia señalada por uno de los profesionales entrevistados consiste en que él, desconocedor del principio de imparcialidad que rige el ejercicio profesional de la interpretación, considera al intérprete profesional como un aliado en comparación con los familiares y amigos que realizan labores de 
interpretación, los cuales, en su opinión, parecen estar del lado del paciente. Esta misma idea se refleja en el estudio llevado a cabo por Angelelli (2008: 150), en el que se pone de manifiesto la presión ejercida por parte de los profesionales sanitarios sobre los intérpretes, a los que se exige en ocasiones que incumplan el principio de imparcialidad. Queda asimismo patente en estudios como el de Leanza (2007: 20), en el que se comenta cómo un gran número de los profesionales sanitarios participantes en la investigación presentada ven al intérprete como un aliado.

Cuando el intérprete es profesional está como de tu lado, y cuando el intérprete es un familiar del paciente, está como en el otro lado y la barrera es mayor. Cuando es un profesional, el intérprete es más como tu aliado. Esa es la única diferencia que veo. [Obstetra 3]

Con respecto a los requisitos necesarios para llevar a cabo labores de interpretación, la mayoría de los profesionales participantes en el estudio opinan que el conocimiento de las dos lenguas de trabajo es suficiente para realizar labores de interpretación.

- Sí, yo creo que con que tengan conocimientos lingüísticos es suficiente, sí. [Anestesista 2]

- Si tiene un buen conocimiento de las dos lenguas, yo creo que sí es suficiente. [Anestesista 3]

- Yo creo que sí, ¿no? Vamos, si sabe español y el otro idioma creo que es suficiente... [Enfermera 4]

- Por supuesto, claro. Por ejemplo, si una señora viene de Marruecos y el intérprete habla árabe y español, pues ya... [Matrona 7]

- Considero que si sabes los dos idiomas perfectamente, puedes hacer de intérprete. [...] Por eso digo, para la cosa digamos... nuestra, médica, hablando así, a mí me basta con que sepa los dos idiomas. [Obstetra 1]

Otros profesionales consideran que los conocimientos lingüísticos no son suficientes y añaden la necesidad de que el intérprete posea también conocimientos culturales, de terminología médica, o del funcionamiento de los servicios sanitarios.

- Tu traductor tiene que conocer muy bien la terminología médica y la técnica que vas a usar para luego saber traducir lo que les estás diciendo. [Anestesista 4] 
- Claro, una cosa es el conocimiento de la lengua, y otra sería el conocimiento que tenga de la... de la cultura, de las costumbres... Y, me imagino, que con ambas cosas pues sí que serviría. [Enfermera 3]

- Hombre, a ver, lo ideal sería que el intérprete tuviera conocimientos a nivel sanitario. Porque a veces tú puedes saber un idioma, hablarlo medio decente, pero a nivel sanitario no saber decir nada. Así que, si los intérpretes pudieran tener también conocimientos a nivel sanitario, pues sería estupendo. [Matrona 4]

- Yo creo que habría que tener conocimientos médicos también, o al menos una noción de cómo funciona el sistema sanitario español, o sea, debería estar bien integrado en lo que es la cultura sanitaria. [Matrona 6]

Echamos en falta entre los requisitos señalados otras habilidades, conocimientos y aptitudes necesarios para que el intérprete desempeñe adecuadamente su labor, tales como el conocimiento de las técnicas básicas de interpretación, la capacidad de análisis y escucha activa, o el conocimiento y la aplicación de los principios éticos inherentes a esta profesión (Angelelli 2007: 63; Del Pozo 2009: 36-37).

Por lo expuesto hasta el momento, podemos afirmar que existe una falta de conocimiento por parte de la mayoría de los profesionales sanitarios que han participado en nuestra investigación sobre las condiciones de trabajo del intérprete médico, así como sobre el papel y las funciones que este deben cumplir para facilitar la comunicación eficaz entre los profesionales sanitarios y las usuarias alófonas.

\section{Conclusiones}

A partir de los datos aportados por los participantes en el estudio presentado, deducimos que son numerosos los casos en los que, pese a que existen servicios de interpretación profesional disponibles, se ha recurrido en el hospital analizado a intérpretes ad hoc para facilitar la comunicación con las usuarias alófonas. En dichas ocasiones es bastante probable que, por los motivos previamente expuestos, estos intérpretes naturales hayan cometido errores de interpretación o hayan incumplido los principios éticos inherentes a esta profesión y, en consecuencia, no habrá resultado posible instaurar una comunicación plenamente satisfactoria con las usuarias alófonas, comprometiendo la calidad de la asistencia sanitaria prestada.

Sin embargo, la mayoría de los profesionales sanitarios que han recurrido al empleo de intérpretes ad hoc afirman estar satisfechos con los resultados 
obtenidos, los cuales, a su parecer, si no han sido óptimos, al menos han resultado aceptables. El problema radica en que, como señala Hale (2010: 46): "la cuestión importante aquí no es si el médico se contenta con un arreglo, sino si el paciente recibe el tratamiento adecuado". El motivo de su satisfacción con la labor de interpretación ejercida por familiares y amigos de las usuarias está en gran medida relacionado con el desconocimiento no solo de las posibles consecuencias que la adopción de esta solución puede implicar (y de los beneficios que conllevaría el recurso a intérpretes profesionales), sino también del papel del intérprete en ámbito sanitario y de los requisitos que este debe poseer para desempeñar con éxito su labor. De hecho, un gran número de los profesionales sanitarios participantes en el estudio considera que es suficiente con poseer conocimientos lingüísticos para realizar una interpretación de calidad. Al respecto, además, subestiman el nivel requerido de conocimientos de las lenguas de trabajo (Mikkelson 1999) y no tienen en cuenta que, tal y como señalan Phelan y Martín (2010: 5), "the ability to speak two languages does not make an interpreter", sino que son necesarios otros conocimientos, habilidades y aptitudes para ejercer adecuadamente esta labor.

En suma, si bien un número considerable de los participantes en el estudio señalan las barreras lingüísticas como uno de los principales problemas a los que se enfrentan en la asistencia sanitaria a usuarias alófonas, no son adecuados los recursos que estos emplean para tratar de superar dichas barreras. Esta inadecuación viene motivada principalmente por el hecho de que, en general, se observa una ignorancia por parte de los profesionales sanitarios de las condiciones de trabajo del intérprete médico, así como del papel y las funciones que este debe cumplir para facilitar la comunicación con las usuarias alófonas. Igualmente, lleva a que algunos profesionales sanitarios participantes no observen grandes diferencias entre recurrir a familiares y amigos para que actúen como intérpretes ad hoc y emplear a intérpretes profesionales. De hecho, la gran mayoría considera que la única ventaja que presentan los intérpretes formados y preparados es que pueden ofrecerles mayores garantías sobre la fidelidad del mensaje transmitido. No mencionan, sin embargo, la importancia de contar con formación para garantizar otros de los principios por los que se rige esta profesión, los cuales, como hemos señalado al analizar los posibles errores e incumplimientos por parte de intérpretes no preparados, constituyen factores diferenciadores entre los servicios prestados por los intérpretes profesionales y por los naturales. Observamos, por tanto, un escaso reconocimiento por parte de los profesionales sanitarios en relación con la labor del intérprete médico y la formación que este necesita para realizar una interpretación de calidad. Así, como afirma Pöchhacker (2004: 166): 
for a practice or occupation to be acknowledged as a profession, it must be perceived to rest on a complex body of knowledge and skills, mastery of which can only be acquired by specialized training.

Para concluir, cabe señalar que, a pesar de que el estudio cuyos datos presentamos en este artículo se centra en un contexto específico y presenta una dimensión reducida, consideramos que contribuye a demostrar la importancia de educar y concienciar a los destinatarios de los servicios de interpretación, en nuestro caso, los profesionales sanitarios, con el fin de fomentar el recurso a intérpretes profesionales. En él se demuestra cómo, a pesar de percibir las barreras lingüísticas como un obstáculo para la comunicación con las usuarias alófonas y, en consecuencia, para ofrecerles una adecuada asistencia, las soluciones adoptadas para superar dichas barreras son en muchos casos inapropiadas, ya que existe un desconocimiento por su parte de las funciones y principios inherentes a la interpretación en ámbito sanitario. En nuestra opinión, los profesionales del ámbito de la salud recurrirían con menos frecuencia a la interpretación ad hoc si fueran más conscientes de la complejidad del rol del intérprete, de las implicaciones negativas que presenta el recurso a intérpretes naturales y de los beneficios que conlleva el empleo de intérpretes formados y preparados. Por consiguiente, como sostienen Bischoff, Loutan \& García (2009: 283-287) es necesario ofrecer formación e información a los profesionales sanitarios, de manera que conozcan las tareas que le corresponden al intérprete, los requisitos que debe poseer para desempeñarlas adecuadamente y la manera más apropiada para relacionarse e interactuar con él. En última instancia, a través de este estudio queda patente el hecho de que, como señala Corsellis (2010: 12-14), los profesionales sanitarios son un grupo influyente en relación con el recurso a intérpretes formados y con el reconocimiento y el desarrollo profesional de esta figura.

\section{Referencias bibliográficas}

ANGELELLI, Claudia V. (2007) "Assessing Medical Interpreters: The Language and Interpreting Testing Project." The Translator 13:1, pp. 63-82.

ANGelelLI, Claudia V. (2008) "The role of the interpreter in the healthcare setting. A plea for a dialogue between research and practice." En: ValeroGarcés, Carmen \& Anne Martin (eds.) 2008. Crossing Borders in Community Interpreting. Definitions and Dilemmas. Amsterdam \& Filadelfia: John Benjamins, pp. 147-163.

BISCHOFF, Alexander \& Patricia Hudelson. (2010a) "Access to Healthcare Interpreter Services: Where Are We and Where Do We Need to Go?" International Journal of Environmental Research and Public Health 7, pp. 
2838-2844. Electronic version: <http://www.ncbi.nlm.nih.gov/pmc/articles/ PMC2922730/>

Bischoff, Alexander \& Patricia Hudelson. (2010b) "Communicating With Foreign Language-Speaking Patients: Is Access to Professional Interpreters Enough?" Journal of Travel Medicine 17:1, pp. 15-20. Electronic version: <http://onlinelibrary.wiley.com/doi/10.1111/j.1708-8305.2009.00314.x/pdf>

BisCHOFF, Alexander; Louis Loutan \& Sofía García. (2009) En otras palabras. Guía para la consulta médica intercultural. Comunicación a través de un intérprete en los servicios de salud. Electronic version: <http://www.universaldoctor.com/ EnOtrasPalabras/>

BRISETT, Camille; Yvan Leanza \& Karine Laforest. (2013) "Working with interpreters in health care: A systematic review and meta-ethnography of qualitative studies." Patient Education and Counseling 91:2, pp. 131-140.

Corsellis, Ann. (2009) Public Service Interpreting: The First Steps. Basingstoke: Palgrave MacMillan. Citado por la traducción española de Carmen ValeroGarcés \& Rosa Cobas: Traducción e interpretación en los servicios públicos. Primeros pasos. Granada: Comares, 2010.

Del Pozo, María Isabel. (2009) "Una propuesta formativa en interpretación en los servicios públicos en el marco del EEES." Redit 3, pp. 31-51.

DiAmond, Lisa C.; Yael Schenker; Leslie Curry; Elizabeth H. Bradley \& Alicia Fernández. (2009) "Getting by: underuse of interpreters by resident physicians." Journal of General Internal Medicine 24:2, pp. 256-262. Electronic version: <http://www.ncbi.nlm.nih.gov/pmc/articles/PMC2628994/pdf/11606_ 2008_Article_875.pdf>

FLORES, Glenn. (2005) "The impact of medical interpreter services on the quality of health care: a systematic review." Medical Care Research and Review 62:3, pp. 255-299.

Flores, Glenn; Milagros Abreu; Cara Pizzo Barone; Richard Bachou \& Hua Lin. (2012) "Errors of Medical Interpretation and Their Potential Clinical Consequences: A Comparison of Professional Versus Ad Hoc Versus No Interpreters." Annals of Emergency Medicine 60:5, pp. 545-553.

Hale, Sandra Beatriz. (2007) Community Interpreting. Basingstoke: Palgrave MacMillan. Citado por la traducción española de Rosa Cobas y Carmen Valero-Garcés. La interpretación comunitaria. La interpretación en los sectores jurídico, sanitario y social. Granada: Comares, 2010.

LEANZA, Yvan. (2005) "Roles of community interpreters in paediatrics as seen by interpreters, physicians and researchers." Interpreting, Special Issue: Healthcare Interaction: Discourse and Interaction 7:2, pp. 167-192.

LEANZA, Yvan. (2007) "Roles of community interpreters in pediatrics, as seen by, physicians and researchers." En: Pöchhacker, Franz \& Miriam Shlesinger (eds.) 2007. Healthcare interpreting. Amsterdam: John Benjamins, pp. 11-34. 
MiKKELSON, Holly. (1999) "Interpreting is Interpreting - Or is It?" Originally presented at the GSTI 30th Anniversary Conference, January 1999, Monterey, California. Electronic version: <http://www.acebo.com/papers/INTERP1. HTM>

MikKelson, Holly. (2003) "Telephone Interpreting: Boon or Bane?" En: Pérez, Luis (ed.) 2003. Speaking in Tongues: Language across Contexts and Users. Valencia: Universitat de València, pp. 251-269.

NÁPOLES, Anna M.; Jasmine Santoyo-Olsson; Leah S. Karliner; Helen O’Brien; Steven E. Gregorich \& Eliseo J. Pérez-Stable. (2010) "Clinician Ratings of Interpreter Mediated Visits in Underserved Primary Care Settings with Ad hoc, In-person Professional, and Video Conferencing Modes." Journal of Health Care for the Poor and Underserved 21:1, pp. 301-317. Electronic version: <http://www.ncbi.nlm.nih.gov/pmc/articles/PMC3576468/pdf/nihms434480. $\mathrm{pdf}>$

Ozolins, Uldis. (2010) "Factors that determine the provision of Public Service Interpreting: comparative perspectives on government motivation and language service implementation." JoSTrans. The Journal of Specialized Translation 14, pp. 194-215. Electronic version: <http://www.jostrans.org/ issue14/art_ozolins.pdf>

Phelan, Mary \& Mayte C. Martín. (2010) "Interpreters and cultural mediators different but complementary roles." Translocations 6:1, pp. 4-20.

Phelan, Mary \& Sue Parkman. (1995) "How To Do It. Work with an interpreter." BMJ 311, pp.555-557.

PÖCHHACKER, Franz. (2004) Introducing Interpreting Studies. Londres: Routledge.

VAlero-GarCÉs, Carmen \& Anne Martin (eds.) (2008) Crossing Borders in Community Interpreting. Definitions and Dilemmas. Amsterdam \& Filadelfia: John Benjamins.

VALERO-GARCÉS, Carmen. (2013) “Comunicación con población extranjera en el ámbito sanitario. Iniciación a una base de datos sobre artículos de investigación." Panacea 37, pp. 80-86. Electronic version: <http://www.medtrad.org/ panacea/IndiceGeneral.htm\#Voll4N37>

WAdENSJÖ, Cecilia. (1998) Interpreting as interaction. New York: Addison Wesley Longman Inc.

WAdEnSJÖ, Cecilia. (1999) "Telephone Interpreting and the Synchronization of Talk in Social Interaction." The Translator 5:2, pp. 247-264. 


\section{NOTA BIOGRÁFICA / BIONOTE}

Almudena NeVAdo Llopis es doctora en Traducción, Sociedad y Comunicación por la Universidad Jaume I de Castellón. Actualmente imparte docencia en interpretación y mediación en el Grado en Traducción y Comunicación Intercultural de la Universidad San Jorge de Zaragoza. Es miembro del grupo de investigación SOCUCO (Sociedad, Cultura y Comunicación) en esta última universidad y de la Red Comunica, Observatorio Permanente sobre la Traducción e Interpretación en los Servicios Públicos.

Almudena Nevado Llopis holds a Ph.D. in Translation, Society and Communication from Jaume I University (Castellón). She is currently lecturer in the Translation and Intercultural Undergraduate Programme of San Jorge University (Zaragoza). She is member of the SOCUCO research group (Society, Culture and Communication) of San Jorge University and belongs to Red Comunica - Permanent Observatory of Public Services Translation and Interpreting. 